\title{
Benzoic acid fermentation from starch and cellulose via a plant-like $\beta$-oxidation pathway in Streptomyces maritimus
}

\author{
Shuhei Noda', Eiichi Kitazono ${ }^{2}$, Tsutomu Tanaka', Chiaki Ogino ${ }^{1 *}$ and Akihiko Kondo ${ }^{1}$
}

\begin{abstract}
Background: Benzoic acid is one of the most useful aromatic compounds. Despite its versatility and simple structure, benzoic acid production using microbes has not been reported previously. Streptomyces are aerobic, Gram-positive, mycelia-forming soil bacteria, and are known to produce various kinds of antibiotics composed of many aromatic residues. S. maritimus possess a complex amino acid modification pathway and can serve as a new platform microbe to produce aromatic building-block compounds. In this study, we carried out benzoate fermentation using S. maritimus. In order to enhance benzoate productivity using cellulose as the carbon source, we constructed endo-glucanase secreting S. maritimus.

Results: After 4 days of cultivation using glucose, cellobiose, or starch as a carbon source, the maximal level of benzoate reached 257, 337, and $460 \mathrm{mg} /$, respectively. S. maritimus expressed $\beta$-glucosidase and high amylase-retaining activity compared to those of $S$. lividans and S. coelicolor. In addition, for effective benzoate production from cellulosic materials, we constructed endo-glucanase-secreting S. maritimus. This transformant efficiently degraded the phosphoric acid swollen cellulose (PASC) and then produced $125 \mathrm{mg} / \mathrm{l}$ benzoate.

Conclusions: Wild-type S. maritimus produce benzoate via a plant-like $\beta$-oxidation pathway and can assimilate various carbon sources for benzoate production. In order to encourage cellulose degradation and improve benzoate productivity from cellulose, we constructed endo-glucanase-secreting S. maritimus. Using this transformant, we also demonstrated the direct fermentation of benzoate from cellulose. To achieve further benzoate productivity, the L-phenylalanine availability needs to be improved in future.
\end{abstract}

Keywords: Streptomyces, Benzoic acid, Endo-glucanase, Cellulose

\section{Background}

In the past few decades, chemicals and fuel production from renewable resources have attracted attention due to global warming and limited supplies of fossil fuels [1-3]. The aromatic series include a large number of industrially important materials, and production of aromatic compounds using microorganisms is an active research area, as well as production of bio-fuel and other building-block compounds [4]. Phenol production using Pseudomonas putida and $p$-hydroxy cinnamic acid pro-

\footnotetext{
*Correspondence: ochiaki@port.kobe-u.ac.jp

'Department of Chemical Science and Engineering, Graduate School of Engineering, Kobe University, 1-1 Rokkodai, Nada, Kobe 657-8501, Japan Full list of author information is available at the end of the article
}

duction using P. putida and Escherichia coli have been successfully demonstrated [5-7].

Benzoic acid is one of the most useful aromatic compounds, and can be converted to terephthalic acid by the Henkel reaction [8], epsilon-caprolactam by the Snia Viscosa process [9], and phenol by a decarbonation reaction [10]. Terephthalic acid is used to make polyethylene terephthalate and aramid, epsilon-caprolactam is a main component of nylon 6 , and phenol is used to make polycarbonate. Benzoic acid is chemically synthesized via an oxidation reaction of toluene in the presence of potassium permanganate; however, this process is energy intensive. Despite its versatility and simple structure, benzoic acid production using microbes has not been reported previously.

\section{Biomed Central}


Streptomyces are aerobic, Gram-positive, mycelia-forming soil bacteria, and are known to produce various kinds of antibiotics composed of many aromatic residues $[11,12]$. Piel et al. characterized the biosynthesis pathway of the polyketide bacteriostatic agent enterocin in the sediment-derived bacterium, Streptomyces maritimus [13]. S. maritimus possess a complex amino acid modification pathway and can serve as a new platform microbe to produce aromatic building-block compounds. Through a process involving $\beta$-oxidation of cinnamoylCoA into benzoyl-CoA, S. maritimus produce benzoylCoA in a plant-like manner from L-phenylalanine during the biosynthesis of the polyketide (Figure 1) [14]. S. maritimus is known to convert benzoic acid to polyketide via benzoyl-CoA using the gene encoding benzoyl-CoA ligase [15]. However, the conversion of benzoyl-CoA to benzoic acid has not been reveled in S. maritimus.

In the present study, we identified $S$. maritimus as a benzoate producer and succeeded in benzoate fermentation using a plant-like $\beta$-oxidation pathway in $S$. maritimus. Bio-production using a biomass resource with Streptomyces as a host has been an area of recent focus due to its great advantages in biomass assimilation [16-18]. In order to examine the carbon-assimilating ability of $S$. maritimus and apply it to benzoate fermentation, we used various carbon sources. We succeeded in benzoate fermentation using glucose, cellobiose, and starch. We compared the carbon-assimilating ability of $S$. maritimus with that of other model Streptomyces, and the versatility of S. maritimus as a host strain was also demonstrated. In benzoate fermentation from cellulose using wild-type $S$. maritimus, the amount of produced benzoate was considerably low, compared to using other carbon sources. The cellulose degradation to glucose and cello-oligosaccharide is one of rate-limiting steps. Here, to enhance benzoate productivity from cellulose, we constructed endo-glucanase (EG)-secreting S. maritimus. The engineered S. maritimus expressed EG from Thermobifida fusca YX and efficiently degraded cellulose. Using this strain, we successfully demonstrated the direct fermentation of benzoate cellulose.

\section{Results and discussion}

Identification and fermentation of benzoate using $S$. maritimus

After cultivation of $S$. maritimus/WT using TSB medium, benzoate produced in $S$. maritimus was identified using a co-chromatography method and UV spectrophotometer $[19,20]$. Figure 2(A) shows a chromatogram of a standard sample of benzoic acid solution (Lane 1) and the culture supernatant of $S$. maritimus/WT containing produced benzoic acid (Lane 2). The peak of benzoic acid in the standard sample was found at about $8 \mathrm{~min}$ (Lane 1), which was also observed in the culture supernatant of $S$. maritimus/WT (Lane 2). The addition of L-phenylalanine into the initial culture medium increased the peak areas of benzoic acid (data not shown). The UV spectra of the benzoic acid fraction separated from the culture supernatant of $S$. maritimus/WT exhibit two major absorption peaks in the region of $190-300 \mathrm{~nm}$, similar to the standard sample of benzoic acid (Figure 2(B)). In addition, we carried out MS spectra analysis. MS and tandem MS analysis show that the peak of benzoic acid dehydrogenated and decarboxylated was observed

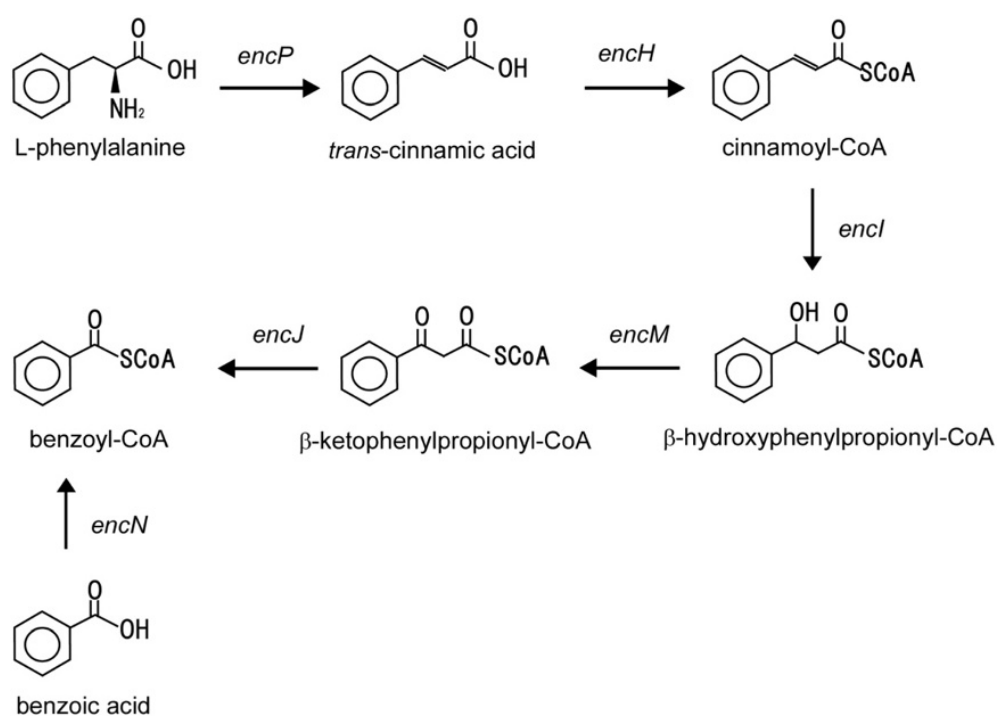

Figure 1 Proposed biosynthesis pathway from L-phenylalanine to benzoic acid. 


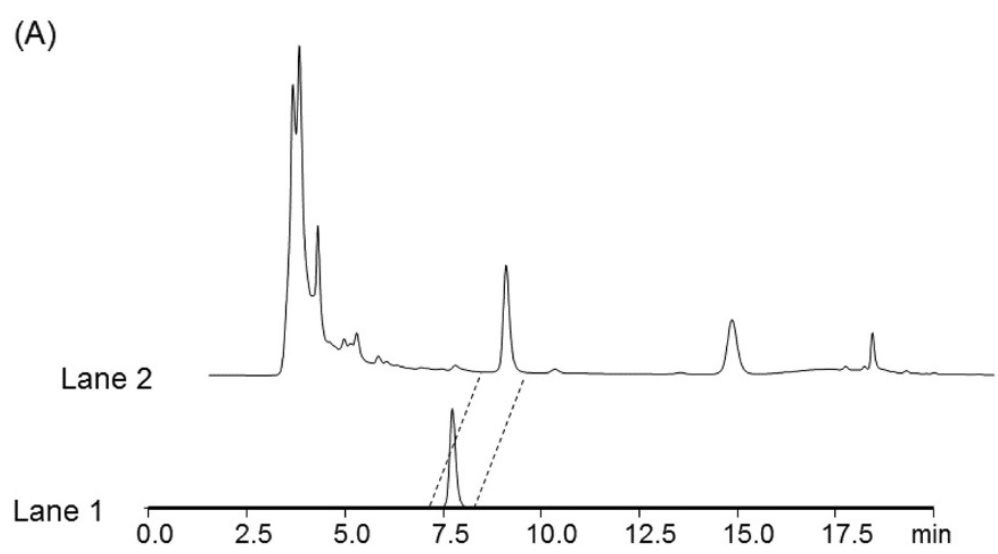

(B)

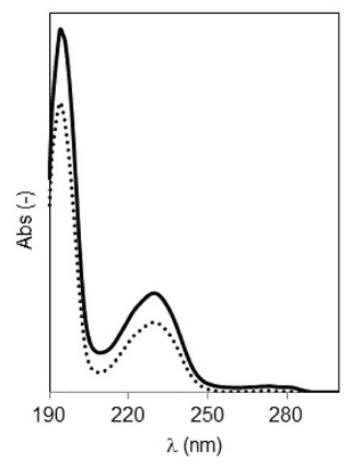

Figure 2 (A) HPLC traces of benzoic acid analysis. Lane 1: Standard sample of benzoic acid in acetonitrile: phosphate buffer (50 mM, pH 2.5) (30:70) solution. Lane 2: The culture supernatant of S. maritimus. (B) UV spectra of benzoic acid. $2.5 \mathrm{mg} / \mathrm{I}$ Standard sample of benzoic acid in acetonitrile: phosphate buffer (50 mM, pH 2.5) (30:70) solution (dotted line), 4 mg/l benzoic acid fraction separated from the culture supernatant of S. maritimus by HPLC (solid line).

around $\mathrm{m} / \mathrm{z} 121.10$ and 77.00 , respectively. These results demonstrated that $S$. maritimus/WT produces benzoate in the culture supernatant.

In order to enhance benzoate productivity, we tested benzoate fermentation using $3 \%$ glucose or xylose as the carbon source using S. maritimus/WT. Figure 3(A) shows time-courses of dry cell weight. S. maritimus/WT consumed glucose or xylose within 3 days (data not shown), and the maximum dry cell weight of $S$. maritimus/WT using xylose was slightly higher than that using glucose (Figure 3(A)). Figure 3(B) shows the amount of produced benzoate. Although it had similar cell-growth ability, the maximal amount of produced benzoate from glucose was $260 \mathrm{mg} / \mathrm{l}$, which was 3-fold higher than that using xylose (Figure $3(\mathrm{~B})$ ). Figure 3 shows benzoate production started after cell growth reached the maximal level. This indicates that the cell growth before benzoate production is one of key factor in benzoate fermentation using $S$.maritimus. In this study, S. maritimus completely consumed $3 \%$ glucose after 3 days cultivation, and the cell of $S$. maritimus reached the maximal level after 3 days. The cell growth is a key factor of benzoate fermentation using S. maritimus. These imply that sugar uptake is one of rate-limiting step in cell growth and benzoate fermentation using $S$. maritimus. In Streptomyces, a lot of the genes encoding sugar transporter were identified. The sugar consumption may be improved by introducing those genes, and the more rapid cell growth and production of benzoate may be achieved. The addition of L-phenylalanine improved benzoate productivity from xylose (data not shown), which indicates that L-phenylalanine availability in $S$. maritimus using xylose as the carbon source is not enough for benzoate production.

In enterocin biosynthesis pathway of S. maritimus, benzoyl-CoA, which is a precursor of benzoate, is converted to enterocin by multiple enzymes. Here, to improve benzoate productivity, we carried out inactivation of encABCL closely concerning enterocin production. We successfully disrupted encABCL in S. maritimus, and the engineered strain was named $S$. maritimus/ $\triangle e n c A B C L$. Using S. maritimus/ $\triangle e n c A B C L$, benzoic acid fermentation was tested. Inactivation of encABCL had no negative effect on the cell growth of $S$. maritimus, however, benzoic acid productivity drastically decreased, compared to wild-type S. maritimus (data not shown). 


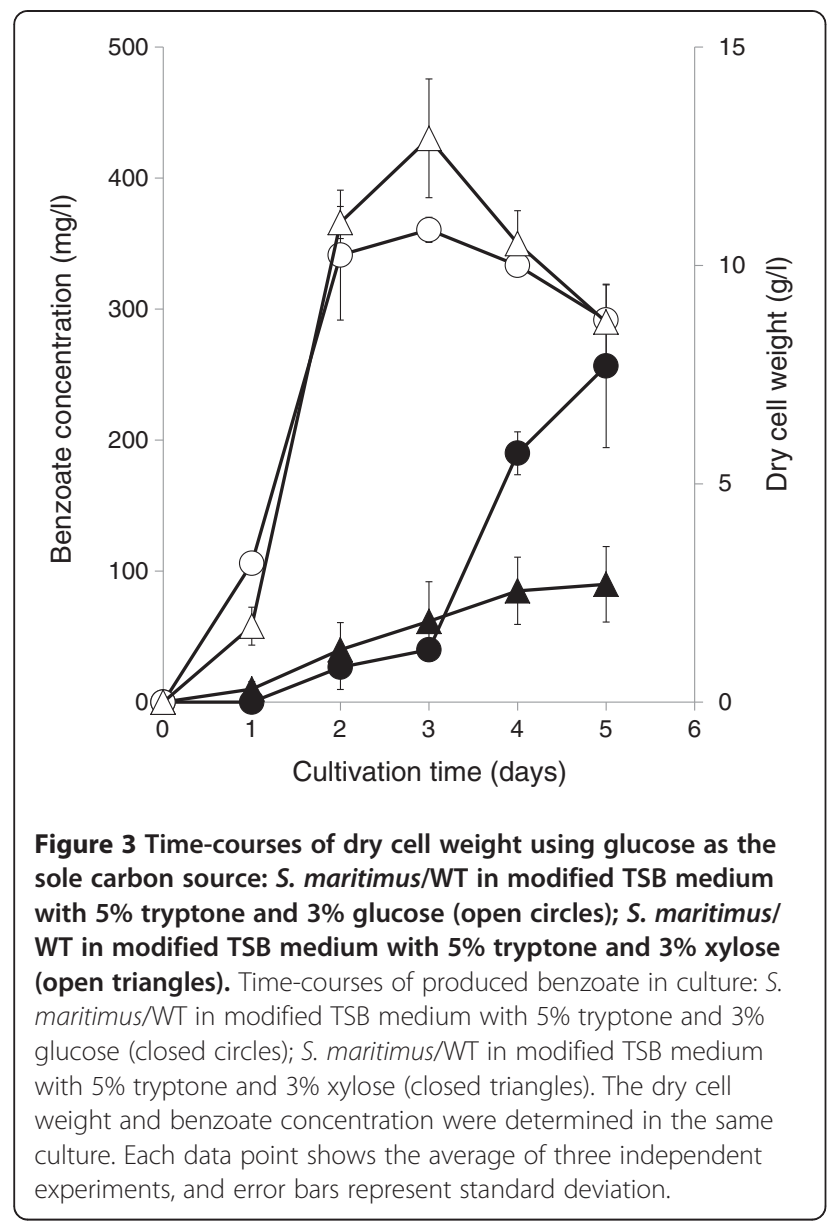

Benzoate production from cellobiose and starch using wild-type S. maritimus

Although some Streptomyces are known to assimilate various carbon sources, such as oligosaccharides and starch $[18,21]$, the carbon-assimilating ability and growth profile of $S$. maritimus have not been investigated.

Figure 4(A) shows the time-course of dry cell weight using $3 \%$ cellobiose, and the maximum dry cell weight of S. maritimus/WT using cellobiose is higher than that using glucose. As shown in Figure 4(B), the maximal level of produced benzoate using $3 \%$ cellobiose was $323 \mathrm{mg} / \mathrm{l}$ after 6 days of cultivation, and the estimated yield for benzoate was $1.76 \mathrm{Cmol} \%$ (Table 1). In the present study, S. maritimus/WT almost completely consumed $3 \%$ cellobiose within 2 days (data not shown). We compared the $\beta$-glucosidase (BGL) activity of $S$. maritimus to that of S. lividans and S. coelicolor, which are model strains in Streptomyces. Figure 4(C) shows the time-courses of BGL activity in the intracellular fractions of S. maritimus, S. lividans, and S. coelicolor. The intracellular BGL activity detected in $S$. maritimus reached 46.1 U/g-drycell, which was 5-fold higher than that in $S$. lividans and S. coelicolor (Figure $4(\mathrm{C})$ ) after 4 days of cultivation. Although the BGL activity expressed in $S$. maritimus was vastly higher than those in S. lividans or S. coelicolor, the cellobiose consumption rates among these strains were almost the same. This result indicates that cellobiose uptake is the rate-limiting step, and that overexpression of the sugar transporter may improve the cellobiose consumption and benzoate productivity in $S$. maritimus. Although the benzoate productivity using xylo-oligosaccharide was lower, similar to that using xylose, the cell growth was similar to that using other carbon sources (data not shown).

We carried out benzoate fermentation using starch. Figure 4(A) shows the time-course of dry cell weight using 3\% starch. The cell growth of S. maritimus using starch as the carbon source was similar to that using glucose (Figure 3(A), 4(A)). As shown in Figure 4(B), the maximal level of produced benzoate using 3\% starch was $460 \mathrm{mg} / \mathrm{l}$ after 6 days of cultivation. The estimated yield of benzoate using starch as the carbon source was 2.64 Cmol\%, which was 1.77 times higher than that using glucose (Table 1). Surprisingly, benzoate production using starch as the carbon source was more efficient than when using glucose. This indicates that $S$. maritimus can directly assimilate starch for benzoate production more effectively than glucose. Although the cell growth of $S$. maritimus using cellobiose was higher than that of using starch, the maximal level of produced benzoate using starch was about 1.5 times greater, compared to using cellobiose. This may indicate that starch as the carbon source encourages the carbon flux to flows smoothly to benzoate synthesis pathway in S. maritimus. Here, to demonstrate that additional carbon sources were used for benzoate formation and the cell growth of $S$. maritimus, we carried out benzoate fermentation in S. maritimus using TSB medium with additional 5\% tryptone (without additional each carbon source). The maximal level of produced benzoate reached about only $100 \mathrm{mg} / \mathrm{L}$, and the cell growth of $S$. maritimus was drastically low, compared to fermentation with additional carbon source. Hence, we conclude that additional glucose, cellobiose, and starch were used for the cell growth, and encouraged benzoate formation.

After the fermentation, the amount of produced cinnamic acid, one of the easily detected intermediates, was $170 \mathrm{mg} / \mathrm{l}$ (from 3\% starch) and $73 \mathrm{mg} / \mathrm{l}$ (from 3\% glucose), respectively. These results show that the ratio of cinnamic acid to benzoic acid ( $\mathrm{mol} / \mathrm{mol}$ ) was increased along with the increased the amount of produced benzoate, suggesting that optimization of the carbon flux may improve benzoate productivity. Enhancing L-phenylalanine availability in the cell may also lead to further benzoate productivity, because the amount of produced benzoate in fermentation using TSB medium with 1.5\% glucose, $1.5 \%$ tryptone, and additional $100 \mathrm{mM}$ of 
(A)

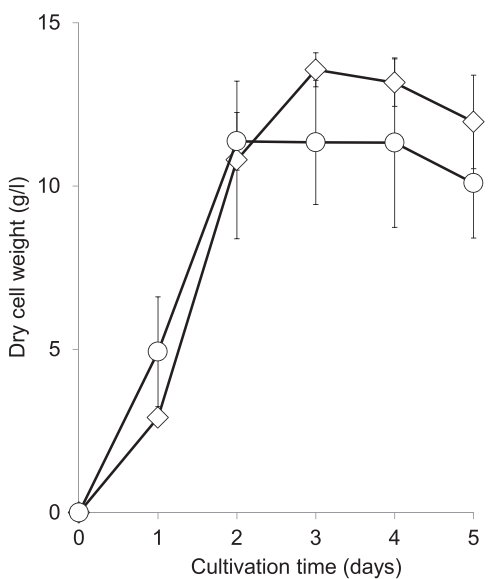

(C)

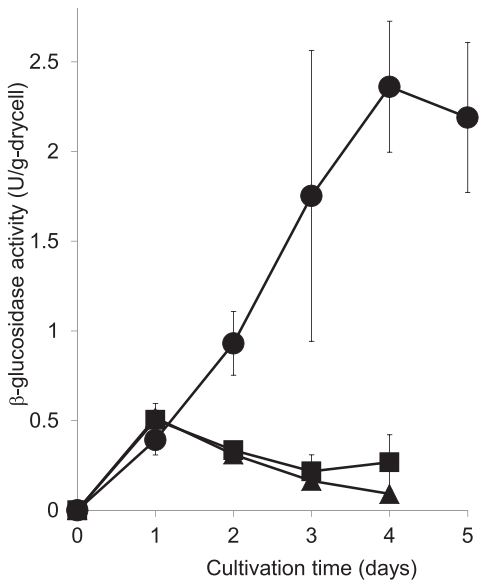

(B)

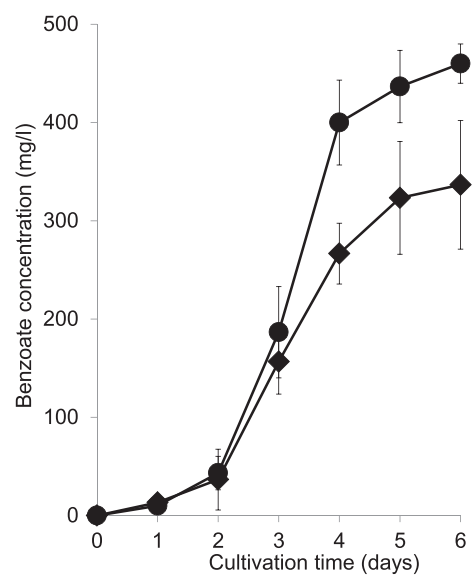

(D)

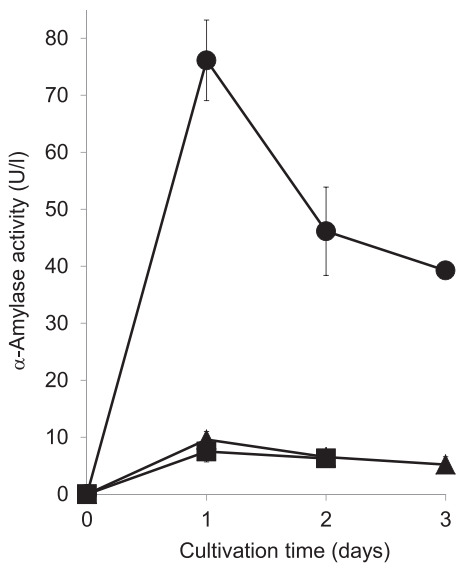

Figure 4 (A) Time-courses of dry cell weight using glucose as the sole carbon source: S. maritimus/WT in modified TSB medium with $5 \%$ tryptone and 3\% starch (open circles); S. maritimus/WT in modified TSB medium with 5\% tryptone and 3\% cellobiose (open diamonds). (B) Time-courses of produced benzoate in culture: S. maritimus/WT in modified TSB medium with 5\% tryptone and 3\% starch (closed circles); S. maritimus WT in modified TSB medium with 5\% tryptone and 3\% cellobiose (closed diamonds). (C) Time-courses of $\beta$-glucosidase activity in the intracellular fractions of S. maritimus/WT, S. lividans, and S. coelicolor using modified TSB medium with 5\% tryptone and 3\% cellobiose: S. maritimus/WT (closed circles), S. lividans (closed squares), S. coelicolor (closed triangles). (D) Time-courses of a-amylase activity in the culture supernatant of S. maritimus/WT, S. lividans, and S. coelicolor modified TSB medium with 5\% tryptone and 3\% starch: S. maritimus/WT (closed circles), S. lividans (closed squares), S. coelicolor (closed triangles). The dry cell weight and benzoate concentration were determined in the same culture. Each data point shows the average of three independent experiments, and error bars represent standard deviation.

Table 1 Various parameters in benzoate fermentation ${ }^{a}$

\begin{tabular}{lllll}
\hline Strain & Carbon source & $\begin{array}{l}\text { Maximum dry } \\
\text { cell weight } \mathbf{( g / l )}\end{array}$ & $\begin{array}{l}\text { Benzoate } \\
\text { produced }(\mathbf{m g} / \mathbf{l})\end{array}$ & $\begin{array}{l}\text { Yield } \\
(\mathbf{C m o l} \%)\end{array}$ \\
\hline S. maritimus/WT & Glucose (3\%) & $10.8 \pm 0.29$ & $257 \pm 62.4$ & 1.47 \\
S. maritimus/WT & Xylose (3\%) & $12.9 \pm 1.36$ & $90.0 \pm 28.9$ & 0.52 \\
S. maritimus/WT & Cellobiose (3\%) & $13.6 \pm 0.52$ & $337 \pm 65.5$ & 1.83 \\
S. maritimus/WT & Corn starch (3\%) & $11.4 \pm 0.88$ & $460 \pm 36.8$ & 2.64 \\
S. maritimus/WT & PASC (1\%) & - & $23.3 \pm 8.17$ & 0.40 \\
S. maritimus/ps-tfu0901 & PASC (1\%) & - & $125 \pm 8.96$ & 2.15 \\
S. maritimus/ps-tfu1074 & PASC (1\%) & - & $103 \pm 0.94$ & 1.77 \\
\hline
\end{tabular}

${ }^{a}$ Values represent means \pm standard deviation for three independent experiments.

${ }^{b}$ mol of carbon involved in produced benzoate per mol of carbon involved in consumed carbon source. 
L-phenylalanine reached $1.3 \mathrm{~g} / \mathrm{L}$. The biosynthesis pathway of L-phenylalanine is strictly regulated by feedback inhibition concerning produced aromatic amino acids. The random mutagenesis by means of $N$-methyl- $N$ nitro- $N$-nitrosoguanidine (NTG) treatment, followed by selection on solid medium containing phenylalanine analogs, can be effective way to obtain the mutants that be insensitive to feedback inhibition. In addition, one transcriptional activator and two regulator proteins have been identified in the polyketide synthesis gene cluster in $S$. maritimus involving genes concerning benzoate production [13]. Overexpression or deletion of those genes may also enhance the yield of benzoate. Figure 4(D) shows the time-courses of $\alpha$-amylase (AMY) activity in the culture supernatant of S. maritimus, S. lividans, and $S$. coelicolor. The AMY activity in the culture supernatant of $S$. maritimus after 2 days of cultivation is more than 8-times higher than that of S. lividans and S. coelicolor.

S. lividans and S. coelicolor are known as model Streptomyces. The carbon source metabolite pathways and genes concerning various biomass degradation enzymes in S. lividans and S. coelicolor have been widely studied $[21,22]$, and they have been used as production hosts for enzymes and chemical compounds [16-18,25]. In this study, we used $S$. maritimus for benzoate production using various carbon sources and demonstrated that $S$. maritimus expresses high BGL- and AMY-retaining activity compared to that of S. lividans and S. coelicolor. Our results may indicate that $S$. maritimus is a new candidate host strain for useful compound production using biomass resources.

\section{Benzoate production from cellulosic materials using EG-secreting S. maritimus}

Many microbes have difficulty degrading cellulose due to its rigid structure. Although we investigated the cellulose-assimilating ability of S. maritimus/WT, the EG activity expressed by $S$. maritimus/WT was not enough to degrade cellulose sufficiently.

For effective benzoate production from cellulosic materials, we selected two candidate EG, Tfu0901 and Tfu1074, from T. fusca YX [23]. The genes encoding Tfu0901 or Tfu1074 were introduced downstream of the phospholipase D promoter region from Streptoverticillium cinnamoneum in the multi-copy type vector pTONA4. For effective secretion of EG, signal peptide sequence from phospholipase D from Stv. cinnamoneum (pld-s) was fused to upstream of those two EG genes, and Tfu0901 or Tfu1074 in over-expressed in S. maritimus, respectively. The supernatants of $S$. maritimus/pstfu0901 and S. maritimus/ps-tfu1074 after 3 days of cultivation were analyzed by western blotting. A band corresponding to Tfu0901 (calculated molecular mass, $46 \mathrm{kDa}$ ) or Tfu1074 (calculated molecular mass, $43 \mathrm{kDa}$ ) was clearly observed (Figure 5, lanes 4 and 5), whereas no band was observed in the case of S. lividans/WT (Figure 5, lane 2) or S. lividans/pTONA4 (Figure 5, lane 3). These results show successful secretory expression of Tfu0901 and Tfu1074 using S. maritimus. We demonstrated that pld-s encourages protein secretion in S. maritimus, as well as S. lividans.

Using S. maritimus/WT, S. maritimus/ps-tfu0901, and S. maritimus/ps-tfu1074 strains, we carried out benzoate fermentation from phosphoric acid swollen cellulose (PASC). Figure 6(A) shows the time-course of benzoate production using 1\% PASC as the carbon source. The maximal level of produced benzoate was 125 or $103 \mathrm{mg} / \mathrm{l}$ after 4 days of cultivation using S. maritimus/ps-tfu0901 or S. maritimus/ps-tfu1074, respectively, whereas $S$. maritimus/WT produced $23.3 \mathrm{mg} / \mathrm{l}$ benzoate. Figure 6(B) shows the time-course of EG activity using $1 \%$ PASC as the carbon source. The maximum EG activities of S. maritimus/ps-tfu0901 and S. maritimus/pstfu1074 were 646 and 224 U/l (Figure 6(B)), respectively. EG activity detected in the culture supernatant of $S$. maritimus/ps-tfu0901 was 3-fold higher than that of $S$. maritimus/ps-tfu1074. The higher activity of Tfu0901 may be attributed to the large amount of expressed Tfu0901, which was confirmed by SDS-PAGE analysis of the culture supernatant (data not shown). The estimated yields of benzoate from $1 \%$ PASC using S. maritimus/pstfu0901 or S. maritimus/ps-tfu1074 were 2.15 and 1.77 $\mathrm{Cmol} \%$, which were 5.4- and 4.4-times higher than that using S. maritimus/WT, respectively (Table 1 ).

\section{Conclusions}

We examined the carbon-assimilating ability of $S$. maritimus, which was greater than other model Streptomyces, and successfully demonstrated direct benzoate fermentation from starch using wild-type $S$. maritimus and cellulose using genetically modified $S$. maritimus. EG-

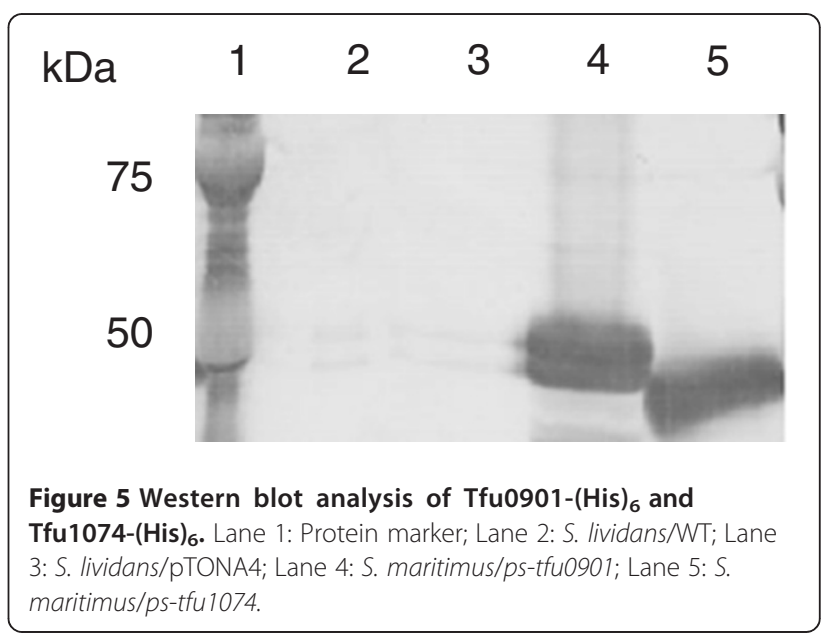



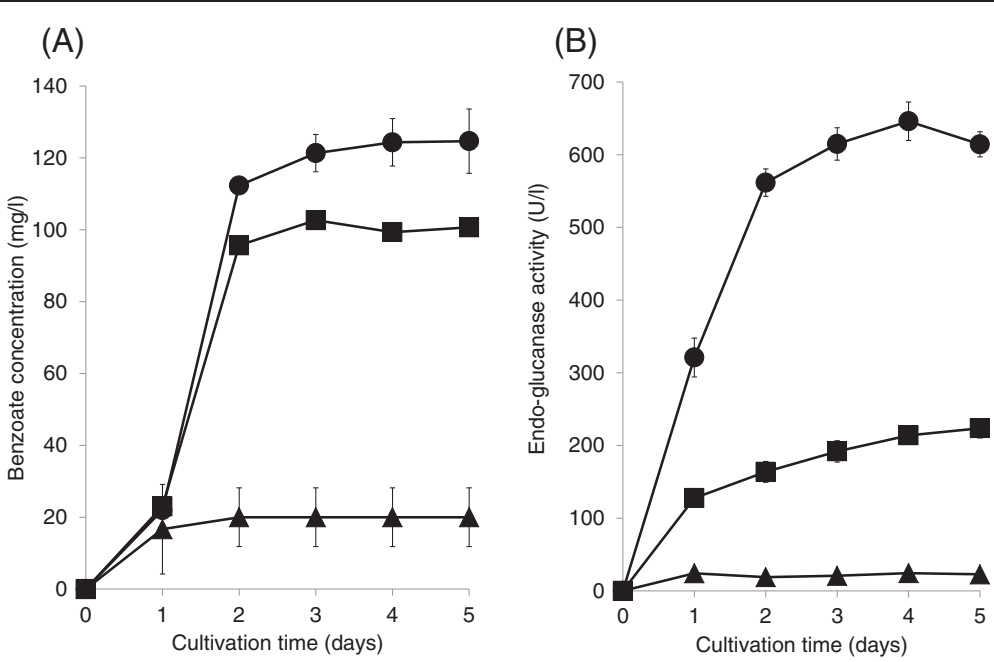

Figure 6 (A) Time-courses of produced benzoate in culture: S. maritimus/ps-tfu1074 in modified TSB medium with $5 \%$ tryptone and $1 \%$ phosphoric acid swollen cellulose (PASC) (closed circles); S. maritimus/ps-tfu0901 in modified TSB medium with $5 \%$ tryptone and $1 \%$ PASC (closed diamonds); S. maritimus/WT in modified TSB medium with $5 \%$ tryptone and $1 \%$ PASC (closed triangles). (B) Time-courses of endo-glucanase activity in the culture supernatant: S. maritimus/ps-tfu1074 in modified TSB medium with 5\% tryptone and 1\% PASC (closed circles); S. maritimus/ps-tfu0901 in modified TSB medium with 5\% tryptone and 1\% PASC (closed diamonds); S. maritimus/WT in modified TSB medium with 5\% tryptone and 1\% PASC (closed triangles). Each data point shows the average of three independent experiments, and error bars represent standard deviation.

secreting S. maritimus efficiently produced benzoate using cellulose just as well as when using glucose. This is the first report concerning benzoate production from cellulose or starch using microbes.

\section{Methods}

\section{Strain and medium}

Wild-type S. maritimus (S. maritimus/WT) was used as the host for benzoate fermentation. For the production of benzoate, a single colony of $S$. maritimus/WT was inoculated in a test tube containing $5 \mathrm{ml}$ of TSB medium [1.7\% pancreatic digest of casein, $0.3 \%$ papaic digest of soybean meal, $0.25 \%$ glucose, $0.5 \%$ sodium chloride, and $0.25 \%$ dipotassium phosphate (BD Diagnostic Systems, Sparks, MD, USA)] supplemented with $50 \mu \mathrm{g} / \mathrm{ml} \mathrm{of}$ kanamycin, followed by cultivation at $28^{\circ} \mathrm{C}$ for 3 days. Then, $5 \mathrm{ml}$ of the preculture medium of S. maritimus/ WT was seeded into a shake flask with a baffle containing $100 \mathrm{ml}$ of modified TSB medium with 5\% tryptone, $50 \mu \mathrm{g} / \mathrm{ml}$ kanamycin, and one of either $3 \%$ glucose, $3 \%$ xylose, 3\% cellobiose, or 3\% cornstarch (Nacalai Tesque, Kyoto, Japan) as a carbon source, followed by incubation at $28^{\circ} \mathrm{C}$ for $5-6$ days.

\section{Plasmid construction and transformation}

Escherichia coli NovaBlue \{endA1 hsdR17( $\left.\mathrm{r}_{\mathrm{K} 12}^{-} \quad \mathrm{m}_{\mathrm{K} 12}^{+}\right)$ supE44 thi-I gyrA96 relA1 lac recA1/F' $\left[\right.$ proAB $^{+}$lacl $^{q}$ $\left.\left.\mathrm{Z} \Delta \mathrm{M} 15:: \operatorname{Tn} 10\left(\mathrm{Tet}^{\mathrm{r}}\right)\right]\right\}$ (Novagen, Inc., Madison, WI, USA), used to construct plasmids, was grown in Luria-Bertani
(LB) medium containing 100 or $40 \mu \mathrm{g} / \mathrm{ml}$ ampicillin or kanamycin at $37^{\circ} \mathrm{C}$.

The vectors for protein expression using S. maritimus as a host and gene deletion of S. maritimus were constructed as follows. The strains and the plasmids used in this study are summarized in Table 2. Polymerase chain reaction (PCR) was carried out using PrimeSTAR HS (Takara, Shiga, Japan).

The promoter and terminator regions of pTONA5 were replaced with Phospholipase D promoter and terminator regions from Streptoverticillium cinnamoneum. The vector was called pTONA4 [24].

The expression plasmids for His-tagged Tfu0901 (Tfu0901-(His) $)_{6}$ ) and Tfu1074 (Tfu1074-(His) 6 ) were constructed as follows. Tfu0901 was amplified by PCR using the Thermobifida fusca YX genome as a template with the following primers: 5'-AAGCTAGCGGTCTCAC CGCCACAGTCACCAAAG-3' (NheI-Tfu0901/Fw) and 5'-TGGATCCTCAGTGGTGGTGGTGGTGGTGGGAC TGGAGCTTGCTCCGCACC-3' (BamHI-Tfu0901/Rv). The amplified fragments were digested with NheI and BamHI and introduced into the NheI and BamHI sites of pUC702-prom-sig-term [25]. The resultant plasmids were called pUC702-ps-Tfu0901-(His) $)_{6}$. The fragments of $p s$-Tfu0901-(His) ${ }_{6}$ and $p s$-Tfu1074-(His $)_{6}$ were amplified by PCR using pUC702-ps-Tfu0901-(His) 6 and pUC702-psTfu1074-(His) ${ }_{6}$ as a template with the following primers: 5'TCGTTTAAGGATGCAGCATGCTCCGCCACCGGCTC CGCCG-3' and 5'-CGCTCAGTCGTCTCAGTGGTGGTG GTGGTGGTGGGACTGGAGCTTGCT-3' or 5'-TCGTT 
Table 2 Strains and plasmids used in this study

\begin{tabular}{|c|c|c|}
\hline Strain, plasmid, or primer & Relevant features & Source or reference \\
\hline \multicolumn{3}{|l|}{ Strains } \\
\hline \multicolumn{3}{|l|}{ Escherichia coli strains } \\
\hline Nova blue & 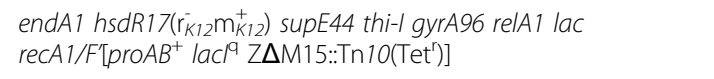 & Novagen \\
\hline S17-1 $\lambda$ pir & TpR SmR recA, thi, pro, hsdR-M+RP4: 2-Tc:Mu: Km Tn7 $\lambda$ pir & BIOMEDAL \\
\hline \multicolumn{3}{|l|}{ Streptomyces maritimus } \\
\hline NT & DSMZ 41777 WT strain & DSMZ \\
\hline /ps-tfu0901 & DSMZ 41777 strain with tfu0901-secreting expression vector & This study \\
\hline \multirow[t]{2}{*}{ Ips-tfu1074 } & DSMZ 41777 strain with tfu1074-secreting expression vector & This study \\
\hline & & This study \\
\hline Streptomyces lividans1326 & WT strain & NBRC \\
\hline Streptomyces coelicolorA3(2) & WT strain & NBRC \\
\hline \multicolumn{3}{|l|}{ Plasmids } \\
\hline pUC702-prom-sig-term & $\begin{array}{l}\text { Versatile vector for protein expression; thiostrepton resistance } \\
\text { marker; pld promoter; rep, replication gene from plJ101; size, } \\
8,600 \mathrm{bp}\end{array}$ & 25 \\
\hline pUC702-ps-Tfu0901-(His) 6 & $\begin{array}{l}\text { Vector for secreting endoglucanase (Tfu0901); thiostrepton } \\
\text { resistance marker; pld promoter; rep, replication gene from } \\
\text { plJ101; size, 9,900 bp }\end{array}$ & This study \\
\hline pUC702-ps-Tfu1074-(His) 6 & $\begin{array}{l}\text { Vector for secreting endoglucanase (Tfu1074); thiostrepton } \\
\text { resistance marker; pld promoter; rep, replication gene from } \\
\text { plJ101; size, 9,800 bp }\end{array}$ & 25 \\
\hline pTONA4 & $\begin{array}{l}\text { Versatile vector for protein expression; kanamycin and } \\
\text { thiostrepton resistance marker; pld promoter; rep, replication } \\
\text { gene from plJ101; size, 9,000 bp }\end{array}$ & This study \\
\hline pTONA4-ps-tfu0901 & $\begin{array}{l}\text { Vector for secreting endoglucanase (Tfu0901); kanamycin and } \\
\text { thiostrepton resistance marker; pld promoter; rep, replication } \\
\text { gene from plJ101; size, 10,300 bp }\end{array}$ & This study \\
\hline pTONA4-ps-tfu1074 & $\begin{array}{l}\text { Vector for secreting endoglucanase (Tfu1074); kanamycin and } \\
\text { thiostrepton resistance marker; pld promoter; rep, replication } \\
\text { gene from plJ101; size, 10,200 bp }\end{array}$ & This study \\
\hline
\end{tabular}

TAAGGATGCAGCATGCTCCGCCACCGGCTCCGCCG -3' and 5'-CGCTCAGTCGTCTCAGTGGTGGTGGTGGT GGTGGCTGGCGGCGCAGGT-3', respectively. Each of the amplified fragments was introduced into the NdeI and HindIII sites of pTONA4 with In-Fusion HD Cloning kit (Takara), respectively [25]. The resultant plasmids were called pTONA4-ps-tfu0901 and pTONA4-ps-tfu1074, respectively.

\section{Intergeneric conjugation and cultivation}

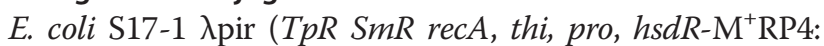
2-Tc:Mu: Km Tn7 $\lambda$ pir) was transformed with each constructed plasmid. A single colony of each transformant was cultivated in $3 \mathrm{ml}$ of LB medium containing $40 \mu \mathrm{g} / \mathrm{ml}$ kanamycin at $37^{\circ} \mathrm{C}$ for $8 \mathrm{~h}$. Cells were harvested, and the cell suspension was washed three times with LB broth and centrifuged to remove kanamycin. The cells were then suspended in $500 \mu \mathrm{l}$ of LB broth and mixed with S. maritimus spores. The mixture was plated on ISP4 medium $(1.0 \%$ soluble starch, $0.1 \% \mathrm{~K}_{2} \mathrm{HPO}_{4}, 0.1 \% \mathrm{MgSO}_{4} \cdot 7 \mathrm{H}_{2} \mathrm{O}, 0.1 \%$ $\mathrm{NaCl}, 0.2 \%\left(\mathrm{NH}_{4}\right)_{2} \mathrm{SO}_{4}, 0.2 \% \mathrm{CaCO}_{3}, 0.0001 \% \mathrm{FeSO}_{4}$, $0.0001 \% \mathrm{MnCl}_{2}, 0.0001 \% \mathrm{ZnSO}_{4}$, and $2.0 \%$ agar). The mixture was then incubated for $18 \mathrm{~h}$ at $30^{\circ} \mathrm{C}$. A 3-ml aliquot of soft-agar nutrient broth containing kanamycin $(50 \mu \mathrm{g} / \mathrm{ml})$ and nalidixic acid $(67 \mu \mathrm{g} / \mathrm{ml})$ was dispensed in layers on the plate, which was then incubated at $30^{\circ} \mathrm{C}$ for $5-7$ days. A single colony was streaked on an ISP4 agar plate containing kanamycin $(50 \mu \mathrm{g} / \mathrm{ml})$ and nalidixic acid $(5 \mu \mathrm{g} / \mathrm{ml})$. The plate was incubated at $30^{\circ} \mathrm{C}$ for $5-7$ days, and selected transformants were named $S$. maritimus/pTONA4, S. maritimus/ps-tfu0901 and S. lividans/ps-tfu1074, respectively.

For production of benzoate, a single colony of $S$. maritimus/ps-tfu0901 and S. maritimus/ps-tfu1074 were inoculated in a test tube containing $5 \mathrm{ml}$ of TSB medium supplemented with $50 \mu \mathrm{g} / \mathrm{ml}$ of kanamycin, followed by cultivation at $28^{\circ} \mathrm{C}$ for 3 days. Then, $5 \mathrm{ml}$ of the preculture medium of S. maritimus/ps-tfu0901 and S. maritimus/ ps-tfu1074 were seeded into a shake flask with a baffle 
containing $100 \mathrm{ml}$ of modified TSB medium with 5\% tryptone, $50 \mu \mathrm{g} / \mathrm{ml}$ kanamycin, and $1 \%$ phosphoric acid swollen cellulose (PASC) as a carbon source, followed by incubation at $28^{\circ} \mathrm{C}$ for $5-6$ days.

\section{Analytical methods}

The benzoic acid and cinnamic acid concentration was simultaneously determined by high-performance liquid chromatography (HPLC; Shimadzu, Kyoto, Japan) using a Cholester column (Cholester residues, $5 \mu \mathrm{m}, 4.6 \times 250 \mathrm{~mm}$, Nacalai Tesque). The operating conditions were $30^{\circ} \mathrm{C}$, with a flow rate of $1.2 \mathrm{ml} / \mathrm{min}$. A dual solvent system was used. Solvent A was phosphate buffer (50 mM, pH 2.5) and solvent $\mathrm{B}$ acetonitrile. The gradient started at $70 \%$ of solvent $\mathrm{A}$ and $30 \%$ of solvent B, A 50-50 mixture of A and B was used from 12 to $17 \mathrm{~min}$. A 70-30 mixture of A and B was used from 17.01 to $20.00 \mathrm{~min}$. The peak of benzoic acid and cinnamic acid in the standard sample were found at about 8 and $12.5 \mathrm{~min}$, respectively. Then, the benzoic acid and cinnamic acid concentration was determined using an ultra-violet absorbance detector (SPD-20AV, Shimadzu). The culture supernatant was separated from the culture broth by centrifugation at $21,880 \times g$ for $20 \mathrm{~min}$, which was followed by analysis using HPLC. UV absorption spectra were obtained using a JASCO V-650 spectrophotometer (JASCO Corporation, Tokyo, Japan). Mass and tandem mass spectra were acquired using 6460 triple stage mass spectrometer (Agilent 6460 with Jet Stream Technology, Agilent Technologies, Waldbronn, Germany) operated at negative ion mode. Voltages for the collision energy and fragmentor voltage were set at 5 and $60 \mathrm{~V}$, respectively. Spectroscopic data of benzoic acid: UV $\lambda_{\max }^{\mathrm{MeOH}} \mathrm{nm}$ : 280; ESI-MS $m / z$ : $121.1\left([\mathrm{M}-\mathrm{H}]^{-}\right)$; ESI-MS/MS $m / z$ (relative intensity(\%)) 121.1(65, [M-H] $\left.]^{-}\right), 77.0\left(100,\left[\mathrm{M}-\mathrm{CO}_{2}-\mathrm{H}\right]^{-}\right)$.

\section{Measurement of biomass degradation enzyme activity}

$\beta$-Glucosidase activity was measured in $25 \mu \mathrm{l}$ of $1 \mathrm{M}$ Tris- $\mathrm{HCl}$ buffer (pH 7.0) with $100 \mu \mathrm{l}$ of $10 \mathrm{mM} p$-nitro phenyl- $\beta$-D-glucopyranoside ( $p$ NPG) (Nacalai Tesque) as the substrate. The mixture (containing $375 \mu \mathrm{l}$ of culture supernatant diluted to $10 \%$ ) was incubated at $30^{\circ} \mathrm{C}$ for $60 \mathrm{~min}$. The reaction was terminated by the addition of $500 \mu \mathrm{l}$ of $3 \mathrm{M}$ sodium carbonate, and the $p$-nitrophenol released was determined by measuring absorbance at $400 \mathrm{~nm}$. One unit of enzyme activity was defined as the amount of enzyme that released $1 \mu \mathrm{mol}$ of $p$-nitrophenol from the substrate per min.

Amylase and EG activity were measured according to a method established by Miller [16], with some modification. A 300- $\mu$ l aliquot of culture supernatant and cell fractions was mixed with $700 \mu \mathrm{l}$ of a $1 \%(\mathrm{w} / \mathrm{v})$ solution of raw starch, which was then dissolved in either $100 \mathrm{mM}$ Tris-HCl buffer ( $\mathrm{pH}$ 7.0) or carboxy methyl cellulose (CMC) dissolved in $100 \mathrm{mM}$ Tris- $\mathrm{HCl}$ buffer ( $\mathrm{pH} 7.0)$.
The mixture was incubated at 37 and $50^{\circ} \mathrm{C}$ for 4 and $1 \mathrm{~h}$, respectively. The amount of reducing sugar released from starch and CMC was assayed by determining the amount of glucose, and equivalents, using the dinitrosalicylic acid method [16]. One unit of enzyme activity was defined as the amount of enzyme that released $1 \mu \mathrm{mol}$ of reducing sugar as glucose, for starch and $\mathrm{CMC}$, and equivalents, from the substrate per min.

\section{Western blotting}

S. maritimus/pTONA4, S. maritimus/ps-tfu0901 and S. lividans/ps-tfu1074 were cultured at $28^{\circ} \mathrm{C}$ for 3 day in $100 \mathrm{ml}$ TSB medium with $3 \%$ cellobiose, 5\% tryptone, and $50 \mu \mathrm{g} / \mathrm{ml}$ kanamycin, respectively. Sodium dodecyl sulfate-polyacrylamide gel electrophoresis (SDS-PAGE) loading buffer was added to the supernatant, followed by boiling at $100^{\circ} \mathrm{C}$ for $5 \mathrm{~min}$. Proteins were analyzed by SDS-PAGE using an SDS-polyacrylamide gel (15\%: w/v), after which proteins were electroblotted onto a polyvinylidene difluoride membrane (Millipore Co., Boston, MA, USA) and were allowed to react with primary rabbit anti-(His) ${ }_{6}$ and secondary goat anti-rabbit immunoglobulin G alkaline-phosphatase-conjugated antibodies (Promega Co., Madison, WI, USA). The membrane was then stained with nitroblue tetrazolium (Promega) and 5-bromo-4-chloro-3-indolylphosphate (Promega) according to the manufacturer's protocol.

\section{Abbreviations \\ EG: Endo-glucanase; BGL: $\beta$-glucosidase; AMY: a-amylase; PASC: Phosphoric acid swollen cellulose.}

\section{Competing interests}

The authors declare that they have no competing interests.

\section{Acknowledgments}

We thank Dr. Maruyama and Dr. Matsuda for helpful discussions and support. This work was supported by Special Coordination Funds for Promoting Science and Technology, from the Creation of Innovation Centers for Advanced Interdisciplinary Research Areas (Innovation Bioproduction Kobe), MEXT, Japan.

\section{Author details}

${ }^{1}$ Department of Chemical Science and Engineering, Graduate School of Engineering, Kobe University, 1-1 Rokkodai, Nada, Kobe 657-8501, Japan. ${ }^{2}$ TEIJIN Holdings USA Inc, 165 Topaz Street Milpitas, New York, CA 95035, USA.

Authors' contributions

S.N. and E.K. designed the experiments. S.N. performed the experiments. S.N and T.T. wrote the paper. C.O. and A.K. commented and supervised on the manuscript. All authors approved the final manuscript.

Received: 27 December 2011 Accepted: 30 April 2012

Published: 30 April 2012

\section{References}

1. Binod P, Sindhu R, Singhania RR, Vikram S, Devi L, Nagalakshmi S, Kurien N, Sukumaran RK, Pandey A: Bioethanol production from rice straw: an overview. Bioresour Technol 2010, 101:4767-4774.

2. Erdei B, Barta Z, Sipos B, Réczey K, Galbe M, Zacchi G: Ethanol production from mixtures of wheat straw and wheat meal. Biotechnol Biofuels 2010, 3:16. 
3. Hong SH, Lee SY: Metabolic flux analysis for succinic acid production by recombinant Escherichia coli with amplified malic enzyme activity. Biotechnol Bioeng 2001, 74:89-95.

4. Gosset G: Production of aromatic compounds in bacteria. Curr Opin Biotechnol 2009, 20:651-658.

5. Nijkamp K. Westerhof RG, Ballerstedt H, de Bont JA, Wery J: Optimization of the solvent-tolerant Pseudomonas putida S12 as host for the production of p-coumarate from glucose. Appl Microbiol Biotechnol 2007, 74:617-624.

6. Vannelli T, Qi WW, Sweigard J, Gatenby AA, Sariaslani FS: Production of p-hydroxycinnamic acid from glucose in Saccharomyces cerevisiae and Escherichia coli by expression of heterologous genes from plants and fungi. Metab Eng 2007, 9:142-151.

7. Wierckx NJ, Ballerstedt $\mathrm{H}$, de Bont JA, Wery J: Engineering of solvent-tolerant Pseudomonas putida S12 for bioproduction of phenol from glucose. Appl Environ Microbiol 2005, 71:8221-8227.

8. Ogata Y, Tsuchida M, Muramoto A: The Preparation of terephthalic acid from phthalic or benzoic acid. J Am Chem Soc 1957, 79:6005-6008.

9. Hirabayashi T, Sakaguchi S, Ishii Y: A new route to lactam precursors from cycloalkanes: direct production of nitrosocycloalkanes or cycloalkanone oximes by using tert-butyl nitrite and $\mathrm{N}$-hydroxyphthalimide. Angew Chem Int Ed Engl 2004, 43:1120-1123.

10. Buijs $W$ : The mechanism of phenol formation in the dow phenol process. J Mol Catal A 1999, 146:237-246.

11. Ikeda H, Kotaki H, Omura S: Genetic studies of avermectin biosynthesis in Streptomyces avermitilis. J Bacteriol 1987, 169:5615-5621.

12. Tamehiro N, Hosaka T, Xu J, Hu H, Otake N, Ochi K: Innovative approach for improvement of an antibiotic-overproducing industrial strain of Streptomyces albus. Appl Environ Microbiol 2003, 69:6412-6417.

13. Piel J, Hertweck C, Shipley PR, Hunt DM, Newman MS, Moore BS: Cloning, sequencing and analysis of the enterocin biosynthesis gene cluster from the marine isolate 'Streptomyces maritimus': evidence for the derailment of an aromatic polyketide synthase. Chem Biol 2000, 7:943-955.

14. Hertweck C, Moore BS: A plant-like biosynthesis of benzoyl-coa in the marine bacterium 'streptomyces maritimus'. Tetrahedron 2000, 56:9115-9120

15. Xiang L, Moore BS: Characterization of benzoyl coenzyme A biosynthesis genes in the enterocin-producing bacterium "Streptomyces maritimus". J Bacteriol 2003, 185:399-404.

16. Ilić SB, Konstantinović SS, Veljković VB, Savić DS, Lazić ML, Gojgić-Cvijović G: Impact of carboxymethylcellulose on morphology and antibiotic production by Streptomyces hygroscopicus. Curr Microbiol 2008, 57:8-11.

17. Kim HS, Park YI: Lipase activity and tacrolimus production in Streptomyces clavuligerus CKD 1119 mutant strains. J Microbiol Biotechnol 2007, 17:1638-1644.

18. Noda S, Miyazaki T, Miyoshi T, Miyake M, Okai N, Tanaka T, Ogino C, Kondo A: Cinnamic acid production using Streptomyces lividansexpressing phenylalanine ammonia lyase. J Ind Microbiol Biotechnol 2011, 38:643-648.

19. Deveryshetty J, Phale PS: Biodegradation of phenanthrene by Alcaligenes sp. strain PPH: partial purification and characterization of 1-hydroxy-2-naphthoic acid hydroxylase. FEMS Microbiol Lett 2010, 311:93-101.

20. González-Barrio R, Truchado P, Ito H, Espín JC, Tomás-Barberán FA: UV and MS identification of urolithins and nasutins, the bioavailable metabolites of ellagitannins and ellagic acid in different mammals. J Agric Food Chem 2011, 59:1152-1162

21. Hurtubise Y, Shareck F, Kluepfel D, Morosoli R: A cellulase/xylanasenegative mutant of Streptomyces lividans 1326 defective in cellobiose and xylobiose uptake is mutated in a gene encoding a protein homologous to ATP-binding proteins. Mol Microbiol 1995, 17:367-377.

22. Tsao LS, Lin LL, Chen JC, Chen JH, Hsu WH: Cloning and characterization of an a-amylase gene from Streptomyces lividans. Biochim Biophys Acta 1993, 1171:255-262

23. Lykidis A, Mavromatis K, Ivanova N, Anderson I, Land M, DiBartolo G, Martinez M, Lapidus A, Lucas S, Copeland A, Richardson P, Wilson DB, Kyrpides N: Genome sequence and analysis of the soil cellulolytic actinomycete Thermobifida fusca YX. J Bacteriol 2007, 189:2477-2486.

24. Hatanaka T, Onaka H, Arima J, Uraji M, Uesugi Y, Usuki H, Nishimoto Y, Iwabuchi M: pTONA5: a hyperexpression vector in Streptomycetes. Protein Expr Purif 2008, 62:244-248

25. Noda S, Ito Y, Shimizu N, Tanaka T, Ogino C, Kondo A: Over-production of various secretory-form proteins Streptomyces lividans . Protein Expr Purif 2010, 73:198-202.
26. Miller GL: Use of dinitrosalicylic acid reagent for determination of reducing sugar. Anal Chem 1959, 31:426-428.

doi:10.1186/1475-2859-11-49

Cite this article as: Noda et al:: Benzoic acid fermentation from starch and cellulose via a plant-like $\beta$-oxidation pathway in Streptomyces maritimus. Microbial Cell Factories 2012 11:49.

\section{Submit your next manuscript to BioMed Central and take full advantage of:}

- Convenient online submission

- Thorough peer review

- No space constraints or color figure charges

- Immediate publication on acceptance

- Inclusion in PubMed, CAS, Scopus and Google Scholar

- Research which is freely available for redistribution 